\title{
LA FUNCIÓN DE BIENESTAR COLECTIVO COMO DECISIÓN RACIONAL
}

\section{Introducción}

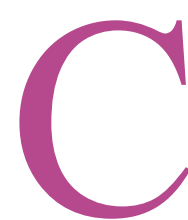
onsiderado dentro del marco de la llamada teoría general del comportamiento racional, el proceso de amalgamar en una única función del bienestar colectivo las preferencias individuales de los distintos miembros de la sociedad sin violentar los principios de coherencia racional, se ve fuertemente impregnado con la reconocida complejidad que exhiben las relaciones entre la racionalidad individual y la racionalidad social.

En efecto, cuando se habla de la racionalidad individual, ya que la misma procura la persecución de preferencias o fines individuales mediante el empleo de los medios más eficientes, no hay dificultad en admitir que se trata de un claro problema de maximización de las utilidades netas de cada individuo. Desde luego que siempre puede ser planteada la cuestión relativa a la calidad diversa de los fines perseguidos, y la eventual discutibilidad de su valor: pero la búsqueda de la maximización de las propias utilidades (relación óptima entre ganancias y costos) permanece siempre como una constante constitutiva del concepto de racionalidad individual. El hecho bastante frecuente de que dos o más «racionalidades» individuales sean completamente distintas y hasta antagónicas, obedece a la circunstancia de que los fines o preferencias planteadas por cada actor también lo son: pero esto de ninguna manera golpea la coherencia del concepto. ya sea cual fuere el fin perseguido y su valor moral: a nivel individual se considera «racional» a quien emplea el medio más eficiente para el logro de un fin. A pesar de que este concepto de racionalidad individual tiene una enorme importancia en numerosos campos, tan importantes como el de la Economía, su capacidad explicativa es más restringida en el terreno de la Ética y la Decisión Política, que son las esferas propias en las que cobra sentido la definición de una función de bienestar social. 
El panorama sereno de la llamada acción racional individual se complica notablemente para el caso de la acción racional en el marco social, ya que en numerosas ocasiones la maximización de las utilidades colectivas entra en conflicto con la racionalidad de los componentes individuales de la sociedad; en virtud de este habitual conflicto, se hace necesario trazar sutiles líneas de articulación entre ambas racionalidades, ya que no es posible evitar la consideración de ninguna de ellas. Con referencia al tema del bienestar colectivo, uno de los aportes más significativos de la teoría general del comportamiento racional, es el de haber logrado establecer una vinculación conceptual coherente entre la racionalidad individual, y la racionalidad en el entorno social [Harsanyi/88].

En el tema específico referido a la construcción de la función de bienestar social, la señalada integración conceptual lograda por la teoría del comportamiento racional ha resultado particularmente fecunda, ya que ha permitido resolver problemas de articulación de funciones individuales sin necesidad de recurrir a la idea de un super-valor. Sin embargo, aún persisten en el campo de la Filosofía del Derecho y de la Filosofía Política algunos desarrollos que inducen a confusiones acerca del alcance y limitaciones metodológicos del enfoque. Tales desarrollos podrían clasificarse en dos grupos: por una parte el de quienes basándose en la imposibilidad (bajo ciertas restricciones) de establecer con procedimientos aditivos una función de bienestar colectivo [Arrow/51] predican la total imposibilidad de construir políticas de bienestar sin vulnerar los intereses y los derechos individuales; y por la otra, la de quienes pretenden que es posible reducir la racionalidad social implícita en las funciones de bienestar colectivo al libre juego de las racionalidades individuales [Gauthier/86].

Por añadidura, el panorama de la discusión acerca de las posibilidades de construir una función de bienestar colectivo en el marco de la decisión racional se torna aún más confuso gracias a quienes, desde una determinada posición, pretenden que las dificultades lógicas y prácticas que la construcción de la función de bienestar colectivo supone, deben ser atribuidas no a la índole extremadamente problemática del asunto a resolver, sino a la debilidad de un enfoque determinado [Dworkin/77].

En el presente trabajo vamos a sostener en primer lugar, que la construcción de la función de bienestar colectivo es una tarea compleja en virtud de que se realiza en tres distintos niveles cuyas estructuras de justificación difieren sustancialmente; por otra parte, estos niveles se vinculan entre sí de un modo tan 
estrecho, que si no se resuelven los problemas de todos y cada uno de ellos no es posible hablar, en sentido ético, de una función de bienestar colectivo.

Para avanzar en este terreno es necesario en primer lugar analizar las distintas formas que pueden exhibir las llamadas decisiones racionales, y el modo en que la acción humana se transforma en virtud de tales decisiones; ya que ubicados en esta plataforma se abre la clara posibilidad de establecer nexos entre la instancia de las decisiones individuales y la de las decisiones éticas, sociales y políticas [Barragán/89-a].

$\mathrm{Al}$ considerar la construcción1 la función de bienestar colectivo como una forma de decisión racional interactiva mediante la cual se dilucida un conflicto entre preferencias, se hace necesario analizar por una parte el viejo problema metodológico acerca de la posibilidad de efectuar comparaciones interpersonales de utilidad, y por la otra, es preciso establecer el escenario específico, dentro de las amplias posibilidades de entorno que ofrece la teoría de la acción racional, al que se adscribe la construcción de una función de bienestar.

Si tal como lo creemos, el problema de la construcción de una función de bienestar colectivo se relaciona con las llamadas decisiones éticas, que se escenifican en el campo de las decisiones racionales en entorno social, se torna imperativo analizar los matices que los clásicos conceptos de la teoría del comportamiento racional adoptan cuando se cumple el arduo proceso de amalgamar múltiples preferencias individuales en una única función de bienestar.

\section{La conflictividad de bienestar colectivo}

El concepto de bienestar colectivo siempre genera debates en el terreno de la Ética, la Política, el Derecho y la Economía. Para algunos se relaciona directamente con el mecanismo básico de que el Estado dispone para superar las inevitables desigualdades existentes entre los hombres. Para otros se encuentra asociado con el peligro que siempre entrañan las restricciones a la autonomía y a los derechos de los individuos de una sociedad determinada. Esta divergencia no resulta sorprendente, ya que en el marco de lo que se entiende por bienestar colectivo, es inevitable dilucidar la espinosa cuestión de qué y cuánto de la propia función de preferencia podemos (o estamos dispuestos) a conceder en favor de una preferencia colectiva, que suele no estar directamente asociada con nuestros propios intereses. Cuando 
se abordan asuntos tan complejos parece muy natural que surjan importantes dificultades tanto conceptuales como metodológicas. Estas dificultades no son simplemente atribuibles a las debilidades de tal o cual enfoque en particular, sino que se derivan de manera necesaria e inevitable del conflicto que se plantea entre las diversas funciones de preferencia individuales. Este carácter conflictivo hace que la función de bienestar colectivo sea siempre restrictiva de uno o varios de los componentes de esas funciones individuales. Puede decirse que trazar límites a las preferencias, derechos e intereses expresados en las funciones individuales es condición de existencia de una función de bienestar colectivo, y resulta natural que quien debe efectuar la renuncia a porciones, a veces importantes, de su propia función de preferencia, tienda a sentirse insatisfecho con las razones que en este sentido le son expuestas. De allí que el decisor político con frecuencia se encuentre enfrentado a un fuego cruzado que tiene su origen en las restricciones impuestas a las diferentes funciones individuales, sin que por lo general cada una de las partes afectadas sea capaz de percibir las restricciones impuestas a otras funciones de preferencia que no sea la suya propia.

En el clásico artículo DeFunis Y. Sweatt [R. Dworkin/77] se plantea un caso sobre la igualdad racial como regla de decisión en una función de bienestar colectivo-, dicho caso evidencia por una parte las dificultades implícitas en este tipo de toma de decisiones y, por la otra, la fragilidad lógica de los esquemas de argumentación normalmente utilizados. Esta fragilidad se deriva de una tendencia que impregna fuertemente el panorama de la Filosofía del Derecho y de la Filosofía Política: se trata de la suposición de que el problema teórico y ético del bienestar es simple de resolver y de justificar coherentemente si se utiliza tal y cual punto de partida, o tal y cual teoría general; sin advertir que en sí misma, como toda decisión que suponía imponer restricciones a la autonomía y a los intereses individuales, la construcción de una función de bienestar colectivo representa un problema terriblemente intrincado, desde cualquier ángulo teórico o ético que se lo aborde. No parece razonable, ni tampoco contribuye positivamente al análisis del problema, el responsabilizar a este o aquel enfoque de una dificultad inherente a la índole del asunto considerado.

El caso en cuestión presenta dos diferentes situaciones de decisión que podrían argumentarse con un mismo esquema, o argumentarse con esquemas antagónicos. La primera situación es la del estudiante negro (Sweatt) que en 1945 se postula para ingresar en la Facultad de Derecho de la Universidad de Texas, 
siendo rechazada su admisión en virtud de una disposición que establecía que sólo los estudiantes blancos tenían derecho a ingresar. Llevado el asunto ante la Corte Suprema, ésta declaró sobre la base de la Décimo Cuarta Enmienda Constitucional, que la ley en cuestión violaba los derechos de Sweatt, ya que la misma establece que ninguna ley estatal puede privar a una persona de la protección igualitaria de sus derechos.

La segunda situación es la de un estudiante judío (DeFunis) que en 1971 solicita su ingreso en la Facultad de Derecho en la Universidad de Washington. El mismo es rechazado, a pesar de haber logrado un score académico superior a todos los negros, filipinos, mexicanos e indios americanos que resultaron admitidos. DeFunis demandó ante la Corte Suprema con el fin de que sobre la base de la misma Décimo Cuarta Enmienda Constitucional, el mecanismo utilizado por la Universidad de Washington para asignar los cupos, consistente en aceptar standards de rendimiento más bajos en los miembros de las minorías étnicas, fuera considerado violatorio de sus derechos.

Las opiniones sobre el asunto DeFunis y Sweatt pueden agruparse en dos diferentes líneas: por una parte, están aquéllas que consideran que ambos casos deben ser justificados desde un mismo esquema de argumentación, y en consecuencia los dos resueltos de una manera idéntica; en este grupo encontramos a la Corte de Primera Instancia que decidió el caso de DeFunis a favor del mismo. El otro grupo, en el que se encuentra Dworkin, sostiene que las bases argumentales deben ser diferentes, y que en consecuencia los casos no pueden ser resueltos en el mismo sentido. Para este grupo las reglas de construcción de ambas funciones de bienestar colectivo no son en manera alguna idénticas, a pesar de que parecen referirse a similares funciones de bienestar individual.

En el análisis del problema, Dworkin ha creído encontrar buenos argumentos que prueban la superioridad del enfoque ideal sobre el utilitarista para capturar la diferencia entre ambas situaciones morales. Según él, en el caso de Sweatt el argumento ideal de una sociedad más justa nos lleva a encontrar la solución correcta al problema; y para que el caso DeFunis pueda tener una solución diferente a la de Sweatt, hay que recurrir también al argumento ideal (el de una sociedad que procura superar injusticias), argumento que en la opinión de Dworkin, derrota en este caso el argumento utilitarista.

Dworkin centra excesivamente su atención en las presuntas debilidades del enfoque utilitarista, por lo que lo que no ahonda en la auténtica naturaleza del problema, que básicamente exhibe 
de manera muy clara la índole conflictiva de todas las funciones de bienestar colectivo. En efecto, en la opinión de Dworkin el criterio racial que lleva a excluir a un estudiante por pertenecer a la raza negra parece no ser aceptable en el caso de Sweatt; pero el mismo criterio racial que lleva a excluir a DeFunis por no ser miembro de las minorías raciales sí le parece aceptable, ya que en este caso el uso de tal criterio tiende a favorecer el ideal «de ser tratado como igual», mientras que en el primero no. Esto prueba que no estamos en presencia de un problema referido solamente al contenido del criterio de decisión, sino también al análisis de otros elementos relacionados por una parte con la naturaleza conflictiva de la función de bienestar, la que siempre lesiona algún interés o derecho, aún en el inocente y probablemente bien inspirado caso de privilegiar a las minorías raciales en su ingreso a la universidad; y, por la otra, con los distintos planos en que la función debe ser resuelta.

El hecho de que Dworkin ofrezca como solución al caso la inexistencia de criterios absolutos y la circunstancia de que todos ellos deben ser verificados respecto al «derecho que tienen todos los miembros de una comunidad a ser tratados como iguales», muestra que no hay fórmulas simples ni generales para resolver la construcción de una función de bienestar colectivo. Pero es evidente, y esto golpearía duramente la posición de Dworkin, que no se avanza demasiado en el camino cuando se remite el problema al «derecho que todos los hombres tienen a ser tratados como iguales», ya que la misma dificultad que se ha hecho presente en la búsqueda del criterio para una «igual selección» surgiría en la determinación de un criterio acerca de lo que debe entenderse por «ser tratado como un igual»; desde luego que la inclusión de lo que Dworkin ha llamado enfoque ideal, en nada contribuye a resolver este problema.

Podrían hacerse algunos comentarios sobre las afirmaciones de Dworkin. En primer lugar, se puede ver que tanto Sweatt como DeFunis no aspiran a ser tratados como iguales, sino como diferentes; y ambos sostienen la validez del criterio diferenciador que más los favorece: en este caso su nivel intelectual; mientras que las minorías raciales de Washington sostienen la validez de otro criterio diferenciador, porque también quieren ser tratadas como diferentes. Lo cierto es que el caso planteado evidencia no sólo un conflicto de intereses individuales, sino también un enfrentamiento entre los valores que constituyen la trama profunda del nivel distributivo de la función de bienestar considerada; y ese conflicto no se resuelve de buenas a primeras sustituyendo el enfoque utilitarista por el ideal, o la expresión 
«ser tratados igualitariamente» por la expresión «ser tratados como iguales».

Por otra parte el caso DeFunis y Sweatt puede ser correctamente argumentado desde cualquiera de los enfoques, tanto a favor como en contra de DeFunis. En efecto, desde la base argumental «Ideal» podríamos sostener el Ideal $1\left(\mathrm{I}_{1}\right)$ : «que todos sean tratados como iguales (no igualmente), admitiendo que esto significa corregir asimetrías previas a la distribución». A partir de la aceptación de $\mathrm{I}_{1}$, y en virtud de que el recurso a asignar es insuficiente, DeFunis no debe ingresar en la Universidad. Pero también podemos sostener el Ideal $2\left(\mathrm{I}_{2}\right)$ : «que todos sean tratados en relación directa con su capacidad». A partir de $\mathrm{I}_{2}$ y siendo el recurso a distribuir limitado, DeFunis sería preferido a cualquier otro con menor capacidad.

Si en lugar de colocarnos en la base argumental «ideal» nos colocamos en la base argumental «utilitaria» y aceptamos la regla Utilitaria $1\left(\mathrm{U}_{1}\right)$ : «maximiza la utilidad esperada de tener una sociedad sin conflictos con la minorías» y considerando lo escaso del recurso a asignar, DeFunis no sería admitido. Sin embargo, si nuestra base argumental es la regla «Utilitarista» 2( $\left.\mathrm{U}_{2}\right)$ «maximiza la utilidad esperada de tener los mejores profesionales», DeFunis, considerando la escasez del recurso, sería preferido. Para el caso de Sweatt, tanto $\mathrm{I}_{1}, \mathrm{I}_{2}$ y $\mathrm{U}_{1} \mathrm{U}_{2}$ permitirían su ingreso. Esto prueba que la argumentación de Dworkin atribuyendo sólo al enfoque utilitarista la inhabilidad para resolver la situación en cuestión, no es válido, ya que ambos enfoques (el Ideal y el Utilitario) son capaces de producir reglas que muestran idéntica ambivalencia cuando se las dota de contenido. Queda asimismo suficientemente evidenciado que un criterio externo inamovible como «el derecho de todos los individuos a ser tratados como iguales» suele convertirse en una fórmula vacía, que no ayuda a esclarecer la situación.

A fin de caracterizar adecuadamente el problema que implica la construcción de una función de bienestar colectivo, es necesario en primer lugar considerar que tal función tiende a producir la distribución de un bien escaso o de una determinada carga social. Tal distribución implica una elección racional que procura articular en una única función las diferentes funciones de bienestar de los individuos afectados por esa decisión. Este plano distributivo (asociado inevitablemente a un conflicto entre intereses) no puede abordarse satisfactoriamente sin tomar en consideración las diferencias y las contradicciones que existen entre las preferencias individuales en juego. Por otra parte, como la función de bienestar colectivo no sólo procura resolver un conflicto 
determinado entre preferencias individuales, sino que también tiene el propósito de ayudar a construir un lenguaje moral de convivencia colectiva, no puede renunciar a su carácter normativo; por esta razón, requiere de una instancia de justificación en la que se discuta extensamente el cuerpo de valores sociales que se propone estimular. En este aspecto la apelación a fórmulas vacías, o a supervalores ideales indiscutibles e inamovibles no consigue generar soluciones aceptablemente validadas.

\section{El Comportamiento Racional}

Dentro del amplio marco de la llamada teoría general del comportamiento racional es posible distinguir una rama que concierne a la teoría del comportamiento racional individual, y otra que se refiere a la teoría del comportamiento racional en el entorno social.

La primera versa sobre las decisiones individuales, las que pueden ser producidas bajo condiciones de certidumbre, de riesgo o de incertidumbre. Se dice que un actor determinado selecciona un curso de acción en condiciones de certidumbre cuando inequívocamente puede predecir el resultado de cualquiera de las acciones susceptibles de ser elegidas; la decisión se efectúa en condiciones de riesgo, cuando el actor conoce la probabilidad objetiva asociada a cada resultado alternativo; $y$, finalmente, la decisión es bajo incertidumbre cuando el decisor sólo se encuentra en condiciones de asignar una probabilidad subjetiva a los posibles resultados. La teoría de la utilidad se ocupa de las decisiones individuales en condición de certidumbre, mientras que la llamada teoría de la decisión se ocupa de tales decisiones bajo las condiciones de riesgo e incertidumbre.

La rama de la teoría general del comportamiento racional que se ocupa de las decisiones racionales en el entorno social se divide a su vez, en la teoría de los juegos, y la teoría ética. Ambas teorías conciernen a toma de decisiones interactivas; pero mientras la teoría de los juegos se refiere al comportamiento de dos o más jugadores que tratan de maximizar racionalmente sus propios intereses individuales, la teoría ética trata con los comportamientos de dos o más individuos que aunque defiendan intereses antagónicos, persiguen como meta común la de maximizar la utilidad general de la sociedad.

En el marco de la teoría del comportamiento racional, se dice que un actor es racional cuando al seleccionar entre los posibles cursos de acción, se inclina por aquél que hace máxima su expectativa 
de utilidad. Esta definición de racionalidad parece funcionar con gran coherencia en el caso de la teoría de la utilidad y en el de la teoría de la decisión, ya que tanto si se conocen ciertamente los posibles resultados, como si se les asignan a los mismos probabilidades objetivas o subjetivas, el decisor está en condiciones de elegir el curso que hace máximas sus utilidades esperadas, sin caer en incongruencia alguna. Pero como la construcción de la función de bienestar colectivo se implanta en el terreno de las decisiones en el entorno social, en ese mismo momento un nuevo conflicto se hace presente: esta vez se trata del enfrentamiento del interés individual con los otros intereses individuales y con el social.

El hecho de que tanto en la teoría de la utilidad como en la de la decisión las acciones racionales exhiban este carácter improblemático no debe sorprender, ya que por definición dentro de ese marco, un actor es racional cuando es capaz de ordenar coherentemente sus preferencias; una acción es considerada racional cuando maximiza las utilidades esperadas, y si se considera que la definición de utilidad se basa también en el ordenamiento coherente de tales preferencias, resulta claro que un actor para ser racional en los términos de la teoría de la utilidad o de la decisión, sólo puede elegir el curso de acción que maximiza sus propias utilidades personales. La teoría económica se ha movido en este ámbito, y por expansión de sus hallazgos, sin duda alguna extremadamente importantes, se ha llegado a pensar que esta racionalidad individual es la racionalidad en términos absolutos; tal creencia ha determinado que en muchas ocasiones se trate a las decisiones racionales en el entorno social, como si fueran conductas racionales individuales, llegando incluso a sostener que es posible construir el nivel ético como una mera adición de decisiones racionales individuales que se corrigen recíproca y espontáneamente en el escenario social (Axelrod/84). En el tema específico del bienestar, este enfoque lleva a sostener que la función de bienestar colectivo se define mediante el libre juego de las funciones de bienestar individual.

Sin embargo en este punto es necesario considerar que aunque las conductas racionales en el entorno social suponen como es natural la presencia de varios actores, y en tales casos cualquier toma de decisión es necesariamente interactiva, mientras en el caso de los juegos cada uno de los actores procura maximizar su propia utilidad individual, en el caso de la ética se procuran hacer máximos los beneficios sociales. Vistas así las cosas, la diferencia entre el planteamiento de los juegos y el de la ética se asocia con la índole (individual o social) de la utilidad maximizada, 
y también con la índole de la interacción. Así mientras en el escenario de los juegos la conducta del jugador oponente es considerada con el solo propósito de trazar estrategias maximizadoras de la propia utilidad, en el terreno de la ética, el comportamiento de los demás miembros de la sociedad es considerado como una información que puede llevar al decisor a cambiar su propio comportamiento, y a trazar límites racionales a su propia función de utilidad. Vistas así las cosas, la auténtica interacción (como disposición a cambiar la propia conducta a la vista del comportamiento del otro) sólo se da en el escenario de la ética.

Es este el otro plano importante a ser tenido en cuenta cuando se intenta la construcción de una función de bienestar colectivo. La armonización de las funciones en conflicto es el objetivo directo o de primer grado de la función de bienestar colectivo; pero el modo en que esas funciones son armonizadas debe estar orientado por la idea rectora de maximización de un valor social que trasciende al plano de la solución del conflicto mismo, para instalarse en el de la credibilidad y permanencia de la trama social toda. Por esta razón, considerada en el marco de la teoría del comportamiento racional, la construcción de la función de bienestar colectivo en un cierto aspecto participa de las características de un juego, con jugadores que como DeFunis y Sweatt hacen sentir la importancia de su función individual, mientras que en otros aspectos se vincula a los rasgos de las decisiones éticas.

\subsection{El problema de las comparaciones interpersonales}

Según se ha visto, en el seno de la llamada función de bienestar se resuelve siempre un conflicto entre preferencias, lo cual supone que en algún momento se ha realizado una comparación entre las mismas; y sobre la base de un cierto criterio se les ha asignado determinado peso dentro de la citada función. Este procedimiento que de hecho se cumple siempre, obliga a formularse preguntas acerca de quién es el que selecciona los criterios a emplear, y acerca de cuáles son las bases sobre las que debe apoyarse tal selección. Preguntas ambas que nos sitúan en el tema fundamental de las comparaciones interpersonales de preferencias.

Este tema de las comparaciones interpersonales de preferencias ha merecido por años (bajo los más diferentes ropajes, y cobijando las más disímiles intenciones) una amplísima atención. 
$\mathrm{Su}$ tratamiento ha resultado en la mayoría de los casos demasiado cargado por muy fuertes emociones e intereses, cosa que en definitiva ha contribuido más a oscurecerlo que a aclararlo. En sí misma la consideración del tema no es nueva, ya que la paradoja a que puede conducir el ordenamiento único de magnitudes no transitivas fue ya considerada con anterioridad. Pero cuando Duncan Black y Kenneth Arrow hacia comienzos de los años 50 realizaron sus trabajos sobre el tema, no sólo avivaron el interés acerca del mismo, sino que, más allá de sus propias intenciones, abrieron de par en par la puerta a los mayores malentendidos. Dos son las principales razones que justifican tal interés, y muy probablemente esas mismas razones son las que estimulan los malos entendidos. En primer lugar, encontramos la simplicidad y elegancia matemática con que ha sido formulada la paradoja, y en segundo lugar, el escenario socio-político con el que se la vincula. Decimos que estas dos razones a la par que han resucitado el interés han contribuido también a desarrollar malos entendidos; porque por una parte, bajo la comprensible fascinación de una construcción matemática impecable se ha llegado a confundirla con un hecho de la propia realidad, y por la otra, porque en el escenario socio-político en que fue colocada la paradoja, en muchas ocasiones se olvida que la misma se encuentra relativizada sólo a las restricciones que la enmarcan (y la hacen existir) y en consecuencia no aspira a reflejar imposibilidad en términos absolutos.

El teorema de Arrow establece que ninguna función de bienestar social puede simultáneamente satisfacer las cinco condiciones por él establecidas. Tales restricciones son las siguientes:

1. El número de elementos será igual o mayor que tres.

2. Asociación positiva de valores sociales e individuales.

3. Independencia de las alternativas irrelevantes.

4. Soberanía del grupo.

5. No dictatorial.

En su formulación alternativa, que es la que mayores malos entendidos estimula, puede decirse que: Si una función de bienestar social satisface las condiciones 1,2 y 3 , entonces o ha sido impuesta externamente al grupo, o es dictatorial. Esta formulación ha sido interpretada en dos sentidos bien diversos: hay quienes la consideran un desafío dirigido a los que creen en la democracia, para que replanteen en forma más coherente sus supuestos; mientras que otras interpretaciones se han inclinado 
por considerarla una clara muestra de la imposibilidad de construir una función de bienestar social sin violentar los valores fundamentales de la democracia.

Pero con el fin de no extraer del mismo consecuencias improcedentes, es necesario considerar que el teorema de Arrow sólo tiene significado en el marco de las restricciones por él propuestas, que definen un mundo extremadamente pobre desde el punto de vista informacional [Arrow/50]; bajo tal supuesto en efecto parece improbable que pueda lograrse una función de bienestar colectivo que sea una transformación lineal de las funciones de bienestar individual (democrática en los términos que se vienen utilizando).

Sin embargo, si miramos algo más allá del formalmente elegante teorema de Arrow, vemos que las decisiones acerca del bienestar colectivo construidas en los estados democráticos toman efectivamente en cuenta y ordenan de alguna manera los diferentes intereses individuales. Este hecho permite al menos poner en tela de juicio la plausibilidad de la construcción de Arrow (que no su coherencia) para describir y analizar el caso concreto de la función de bienestar. Desde luego que para abrir un juicio de plausibilidad es necesario evaluar críticamente los supuestos utilizados, y compararlos con supuestos alternativos. A este respecto se pueden caracterizar nítidamente otras dos posibles vías de acceso al problema que exhiben una excelente tradición: una [Rawls/72] que construye la función de bienestar mediante el ordenamiento de las preferencias individuales y el uso de un criterio externo de decisión (maximin). Este modo de construir una función de bienestar acepta la ordinalidad de las preferencias postulada por Arrow, pero resuelve el problema de comparación mediante la construcción de un orden lexicográfico y una regla maximizadora.

La otra vía de acceso utiliza el supuesto de cardinalidad de las utilidades [v. Neumann-Morgenstern/44]. Desde un punto de vista formal, en el enfoque von Neumann-Morgenstern, si las funciones de utilidad individual son normalizadas respecto de un punto común de referencia, su peso relativo se deriva implícitamente, y desaparece la imposibilidad de construir coherentemente una solución, como lo ha probado Nash con el Dilema del Prisionero [Nash/50]. Desde este punto de vista, excepto en el socialmente infrecuente caso de juegos suma-cero, mediante los métodos de acuerdos y negociaciones, es siempre posible alcanzar una solución coherente que satisfaga suficientemente las funciones de preferencia individuales. En este tipo de enfoque, la posibilidad de soluciones que articulen funciones individuales diferentes, 
o de soluciones que sean transformación de las mismas, es perfectamente válida.

Como puede observarse, fuera de la severa limitación informacional impuesta por las restricciones de Arrow (por otra parte poco defendible en nuestro bien informado mundo) las comparaciones interpersonales pueden efectuarse tanto bajo el supuesto de ordinalidad como de cardinalidad de las preferencias, sin violentar lo que comúnmente se entiende por democracia.

\subsection{La solución en el terreno de los juegos}

Las utilidades de von Neumann han hecho posible un tratamiento algebraico más pleno de las comparaciones interpersonales, y como consecuencia de ello se han desarrollado los llamados mecanismos de negociación y regateo basados en limitaciones racionales recíprocas de las utilidades esperadas, con la finalidad de lograr soluciones socialmente aceptables en términos de utilidad.

Los modelos de regateo se relacionan con la Teoría de los Juegos, que según se señaló está incluida en el campo de la toma de decisión bajo incertidumbre en el contexto social. Recordemos que en tal entorno, los jugadores, a pesar de que interactúan bajo ciertas reglas, lo hacen siempre con el propósito de maximizar su propia utilidad esperada tomando en cuenta las eventuales estrategias del jugador contrincante, pero sin considerar las utilidades sociales. Las reglas que definen el juego son aceptadas por los jugadores, y desde tal punto de vista suponen restricciones a su comportamiento; por otra parte el hecho de que cada jugador ignore cuales serán las respuestas de su contrincante, hace que las decisiones en el marco de los juegos sean siempre bajo incertidumbre; pero en este escenario es claro que los jugadores no asumen ningún punto de vista ético que los lleve a incluir en su decisión la consideración de las utilidades de su adversario.

Sin embargo, la pervivencia del juego como potencial productor de nuevas utilidades se constituye en una forma de valor compartido por los jugadores. Con la excepción de aquel jugador «que siempre pierde», para quien llega un momento en que es preferible no jugar, el juego representa para los jugadores una renovada fuente de potenciales utilidades, y su existencia es algo que merece preservarse.

En relación con la función de bienestar colectivo los llamados juegos no- sumacero- no- cooperativos, que son los más frecuentes 
en la vida social, ofrecen el concepto de «solución» que es extremadamente importante tanto en su aspecto prescriptivo como descriptivo. Considerada normativamente la teoría sugiere a los jugadores una estrategia o conjunto de estrategias (llamada solución) que presenta a los jugadores buenas razones para actuar conforme a ella; y desde el punto de vista descriptivo la teoría provee de una caracterización de las condiciones bajo las cuales puede alcanzarse tal solución. En este sentido la solución fija horizontes y límites a la negociación, definiendo el «espacio posible», y proveyendo de una regla de decisión para escoger el par llamado solución. Formalmente hablando, el concepto de solución para el regateo en el marco de los juegos, sólo consiste en un cuerpo de restricciones y una regla de decisión, que como es natural pueden ser cambiados. En este sentido, dependiendo del enfoque que se tenga del problema, un mismo juego puede tener solución o no tenerla. Sin embargo, el hecho de que los jugadores acepten determinado cuerpo de restricciones los coloca en un marco definido de soluciones posibles; y el hecho de que los jugadores acepten determinada regla de decisión es lo que permite elegir entre las n soluciones posibles, la «solución» que es el par que maximiza las utilidades conjuntas de ambos jugadores.

En un problema de regateo entre dos personas Nash probó en 1950 que bajo cuatro axiomas que definen el marco del juego, existe una única solución a ese problema. El trabajo de Nash fue generalizado, y a veces malinterpretado como una solución al problema de la justa distribución. De los axiomas propuestos por Nash (Óptimo de Pareto, Simetría, Invarianza respecto a transformaciones afines de utilidad e Independencia de Alternativas no-relevantes) este último recibió desde el comienzo importantes críticas. En 1975 Kalai-Smorodinski sugieren el axioma alternativo de monotonía, que nos conduce a otra solución única. Esta es la solución aceptada por Gauthier/86. Las restricciones y la regla de decisión propuestas por Nash, o las propuestas por Kalal-Smorodinski tienen ambas la virtud de reducir las $\mathrm{n}$ soluciones posibles en un problema de regateo (que son $\mathrm{n}$ posibles combinaciones de utilidades de los jugadores) a una «única solución»; pero como es natural, para alcanzar esa única solución, los jugadores han tenido que aceptar desde el comienzo del juego las reglas del mismo, definidas en las restricciones y la regla de decisión. Esta aceptación implica un análisis de las consecuencias que para el resultado del proceso de negociación tiene cada una de las restricciones, y la propia regla de decisión; análisis que como es obvio escapa al acto del juego mismo, y se ubica 
en el terreno valorativo. En consecuencia aceptar o rechazar determinado marco de juego implica abrir un juicio valorativo acerca del posible espacio de soluciones que tal marco genera y de la conveniencia del mismo.

Como puede verse el concepto de solución única dista mucho de ser inequívoco, y desdichadamente no hay reglas formales para resolver el asunto. Desde un punto de vista matemático ambas soluciones (Nash y Kalal-Smorodinski) son perfectamente coherentes; sin embargo, permiten interpretaciones intuitivas muy distintas, lo cual nos impide considerar a alguna de ellas como la solución justa al problema de distribución. [Para el análisis matemático de ambas soluciones, Barragán 89 b]. Esta circunstancia coloca el tema de la selección de la que puede considerarse mejor solución fuera del escenario de los juegos, ya que en realidad pareciera que la suerte de un proceso de negociación queda decidida definitivamente no en el momento del juego mismo, sino al tiempo de construir las reglas que van a regularlo. El fracasado y colosal intento de Gauthier por construir el nivel del bienestar colectivo con el solo auxilio de los procesos de negociación en el marco de la teoría de los juegos, ha contribuido a dejar en claro por una parte, la insuficiencia de ese plano teórico para capturar la complejidad de la construcción de la función de bienestar, y, por la otra, todas las dificultades concretas que en el terreno de la práctica social Y política debe enfrentar el proceso de amalgamar intereses individuales en una única función de bienestar social; pero hay que reconocer que también ha contribuido a caracterizar con mucha claridad la índole conflictiva de la función, y la eventual posibilidad de lograr soluciones negociadas que satisfagan las expectativas conjuntas de los jugadores.

Sin embargo, en el marco de los Juegos no puede encontrar justificación el hecho de porqué preferir una solución a otra, ni tampoco el de cuál es la solución que puede considerarse más justa; de modo que aún cuando el nivel distributivo de la función de bienestar colectivo pueda ser resuelto en este escenario de manera satisfactoria, quedaría aún por considerar el nivel normativo de la misma que se vincula con el logro de soluciones tales que fortalezcan los nexos fundamentales de la vida social.

3.3 La función de bienestar social como transformación lineal de las funciones de bienestar individual

El supuesto de von Neumann acerca de la cardinalidad de las 
utilidades, es consistente con lo que se denomina decisor racional plenamente coherente, es decir aquél que es capaz de ordenar sus alternativas de acción asignándoles un peso relativo en una misma escala de utilidad. La construcción de von Neumann exhibe una notable importancia en dos sentidos: por una parte logra captar analíticamente el procedimiento que de manera intuitiva sigue un decisor racional frente a posibles alternativas de acción; y desde el punto de vista normativo ofrece razones suficientes para que los decisores acepten, practiquen y refinen tal procedimiento. Resulta claro que el problema de las comparaciones intrapersonales queda teóricamente resuelto, ya que cada decisor elabora su propia escala de utilidades, asigna en la misma peso relativo a sus preferencias, y decide en consecuencia. Este enfoque resuelve el problema de construcción de las funciones de utilidad individual de una manera elegante desde el punto de vista formal, y además provee de una base argumental razonable al decisor individual.

Para llegar a construir la función de bienestar colectivo sobre la base de tales preferencias individuales en el marco de este enfoque, es necesario apelar a un nuevo principio que no está presente en los niveles de acción racional que hemos considerado en la teoría de la utilidad, la de la decisión y la de los juegos; se trata del principio «los juicios morales deben ser imparciales», principio que se concreta en las dos reglas de construcción de la función de bienestar colectivo: la primera se refiere a que tal función debe basarse en la maximización de la utilidad social, y la segunda en que la utilidad social es una función lineal de todas las utilidades individuales si se les asigna a cada una de estas el mismo peso relativo. La primera regla podría considerarse una transformación o desarrollo de la regla general de maximización; pero la segunda incorpora expresamente un elemento equiprobalístico que junto con el tipo de interés que considera a la primera regla genera una restricción, que muy bien puede resultar incoherente con el principio de maximización individual.

Asimismo este modelo equiprobabilístico aunque formalmente coherente y en extremo elegante, no logra sortear las dificultades que se presentan al momento de su implementación en la práctica social. En efecto, en primer lugar los individuos no sólo tienen preferencias (como medida de utilidad), sino también tienen ideas acerca de tales preferencias, y les asignan prioridades en diferentes grados; y todo eso no puede explicarse sólo en relación con las constantes más o menos generales de la naturaleza humana, sino básicamente con sus propias historias individuales y entornos culturales. Algunas de esas preferencias serían 
fácilmente aceptables desde cualquier ángulo de interés colectivo; pero respecto de otras, el panorama se torna mucho más dudoso. Surge de esto una pregunta necesaria: ¿qué preferencias deben ser tomadas en cuenta para construir la función de bienestar social? La respuesta que se ha intentado a este problema es la de elaborar ciertas reglas que permitan eliminar determinadas preferencias [Hare/81 y Harsanyi/88]. Las preferencias que se pretende eliminar son las que puedan calificarse de desinformadas, equivocadas, o hetero-orientadas (entre estas últimas tienen especial relevancia las llamadas malevolentes).

Este proceso de eliminación, aparentemente simple, conlleva dificultades teóricas y empíricas gigantescas. En el caso más simple, que es el de las preferencias desinformadas, todo parece reducirse a la eliminación de aquéllas que se apoyan en información incompleta o errónea; pero no podemos olvidar que el proceso de transformar datos en información es variable de un individuo a otro, y de una circunstancia a otra [Barragán 87]. Teniendo en cuenta esto, lo que pueda considerarse como una preferencia "desinformada» depende de criterios subjetivos muy difícilmente comparables, a partir de los cuales las opiniones pueden diferir considerablemente.

Este argumento que acabamos de utilizar para destacar los problemas que surgen cuando se pretende hablar de preferencias desinformadas cobra aún mayor fuerza si se lo aplica a las preferencias «equivocadas». Aquí se une la valoración a la subjetividad ya señalada; y el solo proceso de clasificación de las preferencias eliminables por «equivocadas», supone una decisión que no vacilaríamos en calificar de moral, y que desde el comienzo fija qué preferencias pueden ser tomadas en cuenta, y cuáles no.

Respecto de las preferencias hetero-orientadas o externas en lenguaje de Dworkin, Harsanyi logra probar formalmente que la función de utilidad auto-orientada ( $\mathrm{y}$ en consecuencia la heteroorientada) de una determinada persona $\left(V_{l}\right)$ queda bien definida cuando su función de utilidad completa $\left(U_{l}\right)$, que está formada por las preferencias auto-orientadas y las hetero-orientadas, tiene límite superior finito. Incluso con una determinada restricción esto es válido para toda $U_{l}$. La formalización de Harsanyi resuelve el problema de eliminar las preferencias hetero-orientadas de la función de utilidad completa $U_{I}\left(X_{l}, Y_{l}\right)$, tanto en el caso simple de una función de utilidad separable:

como en el más arduo en que $U_{1}$, no es separable:

$$
U_{l}\left(\mathrm{x}_{1}, \mathrm{Y}_{1}\right)=V_{l}(\mathrm{Xi})+\mathrm{Z}_{1}\left(\mathrm{Y}_{1}\right)
$$




$$
\begin{gathered}
V_{l}\left(\mathrm{x}_{1}\right)=\operatorname{Max} U_{l}\left(\mathrm{x}_{1}, \mathrm{Y}_{1}\right) \\
\mathrm{Y}_{1}
\end{gathered}
$$

es decir, que la función de utilidad autorientada de una determinada persona $V_{l}\left(\mathrm{x}_{1}\right)$, es el nivel de utilidad que i disfrutaría en una determinada posición $\left(\mathrm{x}_{1}\right)$, si todas sus preferencias heteroorientadas fueran máximamente satisfechas.

La duda que persiste es si durante el proceso que lleva a efectuar esas eliminaciones hemos utilizado sólo la apelación inicial a la imparcialidad de los juicios morales, o hemos empleado también puntos de vista moral inevitablemente implícitos en tal proceso. La construcción que sirve de base para la eliminación o reubicación de una determinada preferencia es la llamada «hipótesis de la preferencia bien informada». Esta construcción, como toda hipótesis, se edifica necesariamente sobre argumentos de base muy variada: teórica, empírica, valorativa (por mencionar sólo las principales) todas las cuales contribuyen a definir la hipótesis en cuestión [Barragán 83]. De esto se deduce que no hay hipótesis que reflejen de manera espontánea, objetiva e indudable, los hechos de la realidad; sino que las mismas son enfoques relativizados al punto de vista de quien las produce, a la época en que se producen, y representan siempre solamente juicios acerca de la realidad.

Parece evidente que los elementos presentes en la «hipótesis de la preferencia bien informada» son difícilmente expresables con el sólo apoyo del principio inicial de imparcialidad de los juicios éticos, y en consecuencia demandan la consideración de otros niveles de la función de bienestar colectivo: los vinculados con los juicios de valor que hacen posible no sólo una distribución de recursos sino que además persiguen sostener la estructura general de la convivencia social. Para mantener la coherencia de su formulación, quienes sostienen que la función de bienestar colectivo es una transformación lineal de las funciones de bienestar individual si se asigna igual peso relativo a todas las preferencias, han apelado al recurso de trasponer el problema valorativo, ubicándolo, un tanto solapadamente, en el momento de la definición de la «hipótesis de las preferencias bien informadas»; hipótesis que a su vez decide acerca de cuáles son las preferencias que deben ser consideradas en la función de bienestar, dónde deben estar ubicadas, y desde luego, qué preferencias deben ser excluidas. Después de tal decisión acerca de cuáles son las preferencias que deben ser consideradas, y sólo después, viene el momento de asignación de igual peso relativo a cada una de ellas. 
Este procedimiento que visto así, luce un poco tautológico, expresa claramente que de manera inevitable, y en algún momento del proceso, debe efectuarse la consideración del nivel valorativo; y prueba también que el hecho de poseer una regla instrumental única para la construcción de la función de bienestar colectivo no es una condición suficiente para la existencia de la función de bienestar colectivo en un sentido ético político.

Tal como vemos, hay dos aspectos muy importantes que el enfoque de la función de bienestar colectivo como transformación lineal de las funciones de bienestar individual ha contribuido a esclarecer: el primero muestra la posibilidad cierta de elaborar una regla de decisión sin necesidad de apelar a un super-valor al que tal regla se ligue intrínsecamente. Este tipo de regla ofrece la ventaja de que permite la posibilidad de un acuerdo (que como tal es plural) basado en la conveniencia de que una determinada preferencia sea incluida o no en la función de bienestar colectivo con tal o cual intensidad. El segundo aspecto se relaciona con el hecho de que tal regla por sí sola no puede resolver el problema de asignación de prioridades, pesos y posibles combinatorias, problema que está en la base de la definición de preferencia, noción crucial para la aplicación de la regla de transformación lineal.

Los logros de este enfoque han contribuido a mejorar la comprensión de la naturaleza compleja de la función de bienestar colectivo: por lo que se ve la elaboración de una regla instrumental es condición necesaria para la construcción de esa función; pero para abordar el problema de distribución de bienes y recursos implícito en la misma, se necesita superar el plano instrumental con el fin de atacar la solución del conflicto sustantivo.

Por lo que hemos visto hasta el momento, el tipo de reglas empleadas por los enfoques analizados se han mostrado insuficientes para capturar aspectos cruciales en la construcción de la función de bienestar colectivo. Tanto Rawls con su orden lexicográfico y su regla de decisión maximin, como Nash y KalalSmorodinski con su regla para definir una única solución, o Harsanyi con la regla de linealidad, sólo difieren o desplazan el problema de asignación de los recursos, los bienes o los servicios, que es el problema sustantivo del bienestar. Debemos reconocer, sin embargo, que todos estos enfoques han contribuido de una manera indiscutible a un tratamiento riguroso del problema en el terreno de la ética política y jurídica. 


\section{El plano distributivo de la función de bienestar colectivo}

El análisis del carácter distributivo (y en consecuencia conflictivo) de la función de bienestar colectivo, que por mucho tiempo ha sido acaparado por los economistas, tiene un significado muy especial en el campo de la Filosofía Política y Jurídica; y sin su consideración todo enfoque resulta necesariamente incompleto y ambiguo. Sin embargo, lo cierto es que no resulta fácil para el pensamiento filosófico tener que aceptar que el sistema o código moral de una determinada sociedad sea insuficiente para derivar de manera espontánea e inequívoca los principios de distribución necesarios para esa sociedad. A pesar de esto resulta evidente que los problemas de adjudicación de recursos que constituyen el punto central que la función de bienestar colectivo tiende a resolver, se escapan a las reglas de decisión que hemos analizado hasta el momento, y deben ser considerados desde una perspectiva analítica diferentes. En recientes trabajos algunos filósofos han revitalizado el interés por los problemas de distribución vinculados con la función de bienestar colectivo, tratando de encontrar reglas que permitan resolver las contradicciones inherentes a todo proceso distributivo.

Brandt/89 a pesar de que sostiene que en el sistema moral no se tendría que incluir ningún principio distributivo, ya que éstos deberían ser deducibles del contenido del propio sistema moral, se ha visto obligado a incluir cuatro principios respecto de la distribución del ingreso, los que a su juicio garantizan una función de bienestar colectivo moralmente justificable. Tales principios son: primero, el ingreso real debe ser igual para todos los miembros de la sociedad; segundo, habrá incentivos para cubrir necesidades especiales; tercero, habrá incentivos y recompensas según el tipo de ocupación desempeñada; y cuarto, habrá variaciones para estimular el logro de metas socialmente deseables.

Como puede notarse, Brandt considera al ingreso como el factor distribuidor de bienestar, y a través del mismo procura solucionar el problema de las desigualdades generadas por la existencia de necesidades especiales o de diferenciales en el trabajo. Asimismo reserva un margen para estimular el logro de objetivos deseables desde el punto de vista social.

Vamos a dejar de lado, por el momento, la consideración de las distorsiones que pueden originarse en el enfoque general de Brandt al considerar el ingreso como factor distribuidor de bienestar, ya que escapa al interés presente [ver Walzer/83]. En lo sustancial, aunque con algún disgusto Brandt acepta la imposibilidad de que un sistema moral determinado resuelva el problema 
de la función de bienestar colectivo sin la presencia de algunos principios de distribución. Sin embargo, es necesario hacer notar que su análisis da por supuesto de manera evidente la existencia de una determinada forma moral a la cual es posible asociar los principios distributivos que él señala. Es claro que tales principios distributivos sólo tienen sentido en el marco de un específico sistema moral, y son perfectamente insostenibles respecto de otros. Por otra parte, a pesar de que Brandt usa la expresión «principios» de distribución, lo que él está derivando de un sistema moral específico (en el que el monetarismo tiene sentido) son reglas únicas de distribución del ingreso, cuya finalidad es la de dirimir específicamente los conflictos entre quienes disputan en esa distribución.

Griffin/86 es más explícito al considerar la importancia del problema de distribución en la construcción de la función de bienestar colectivo, colocándolo como un elemento necesario en todo sistema moral; y aunque el marco que utiliza para la consideración de los bienes que tendrían que estar incluidos en esa función es el de la sociedad industrial avanzada, destaca que tanto los bienes a incluir como la regla a utilizar deben hacerse cargo del tipo de estructura de los grupos, de las distintas clases de recursos disponibles, y de la naturaleza específica de la estructura económica a la que se refieren.

Griffin considera acertadamente que los principios distributivos no son principios filosóficos, sino que entrañan elementos muy diversos, y estipula como único lineamiento-guía en la definición de los principios, la consideración de la base sobre la que descansan, y de los mecanismos de aplicación y estructuración de los mismos. Para Griffin habría principios basados en derechos, entre los que coloca los relativos a las provisiones mínimas materiales para llevar adelante el plan de vida; también ubica aquí lo relativo al uso seguro de tales bienes, y al disfrute de la libertad (no considerada en general sino de manera específica) necesaria para vivir el plan de vida. Como puede verse, los principios basados en derechos giran alrededor del llamado plan de vida, lo cual supone ampliar el escenario de la regla de maximización. En cuanto a los principios basados en la igualdad, propone la igual distribución de bienes que incluyen tanto bienes como servicios, y de bienestar para satisfacer las necesidades básicas.

Resulta claro que en el caso de Griffin, el problema de la distribución merece una consideración cuidadosa, y podríamos coincidir con algunas de sus afirmaciones; pero de nuevo lo que comienza siendo un proceso de definición de principios, termina 
convirtiéndose en la búsqueda, y elaboración de reglas generales de distribución. El problema de apelar a reglas de decisión más o menos rígidas, es que las mismas carecen de la versatilidad necesaria para adaptarse a la pluralidad de escenarios conflictivos en que se desenvuelve el problema de la distribución cuando se construye una función de bienestar social, y en consecuencia tienden a duplicar la acción de las reglas instrumentales. El momento de decidir acerca de la distribución de un recurso escaso (único marco en el que la función de bienestar colectivo tiene sentido) no puede abordarse en el mismo plano en que se desarrolló la elaboración de la regla instrumental, porque implica una estructura de justificación que debe hacerse cargo del enfrentamiento concreto, que se produce en un entorno bien preciso, entre las preferencias individuales antagónicas que aspiran a ser resueltas en el marco de significados del bienestar colectivo.

El escenario de decisión racional que más se hace cargo del carácter conflictivo del momento de la distribución de un recurso escaso es el de la Teoría de los Juegos. En tal marco queda perfectamente claro que todos los jugadores implicados en la decisión (incluidos quienes institucionalmente están a cargo de construir la función de bienestar colectivo) procuran maximizar sus propias utilidades; y para lograrlo, efectúan todos los manejos estratégicos que puedan favorecerlos. Como en cualquier juego, la diferencia de habilidad y poder entre los jugadores tiene una gran importancia en el eventual resultado final; y también como en cualquier juego los jugadores tienden a ser mucho más generosamente tolerantes con sus propios artilugios que con los de sus oponentes. Sin embargo, si nos ubicamos en el caso socialmente más frecuente que es el de los juegos- nosuma -cero -no -cooperativos, también es cierto que los jugadores están unidos por un mínimo de interés común que es su deseo de que el juego continúe, ya que sus utilidades esperadas dependen de este hecho.

Del mismo modo que en un juego, mediante el solo recurso de la distribución no hay manera de garantizar un determinado resultado, ya que todos los jugadores son libres de administrar, y hasta de dilapidar sus propios recursos y posibilidades. Por esa razón considero que no es una vía fecunda de acceso al problema de la búsqueda de reglas rígidas de asignación. Como en todos los juegos, el juego social debe ser interesante, y para serlo ha de tener un marco insesgado a fin de que el resultado final no sea tan claramente previsible como para tornarlo tedioso o francamente desventajoso para cualquiera de los jugadores. En este entorno, mientras los jugadores por una parte procuran estratégicamente 
lograr marcos que les permitan sacar el mejor partido de su propia situación y de sus condiciones específicas, por la otra, también estratégicamente, buscan que se preserven las utilidades derivadas de la conservación de un juego interesante.

Hasta aquí parece satisfactoriamente argumentada la imposibilidad de que se logren reglas distributivas que por sí solas sean garantía de resultados justos. Esto es así, porque la distribución misma es un hecho inevitablemente estratégico, que llega a privilegiar determinados rasgos diferenciales en detrimento de otros; y por lo demás esas reglas serán luego estratégicamente utilizadas por los jugadores. También parece que lo máximo a que puede aspirarse en este escenario es a dotar al juego de un marco insesgado. Esto puede lograrse mediante la construcción interactiva del mismo; pero la interacción aunque garantice satisfactoriamente la ausencia de sesgos, no es suficiente garantía de que el marco sea el más aceptable socialmente. Esto último da lugar a una pregunta que no puede encontrar respuesta en el plano distributivo, sino en el plano normativo de la función de bienestar colectivo. Solamente en esa instancia es posible elaborar una justificación suficiente que permita hablar de una función socialmente aceptable en razón de su justicia; y solamente con la inclusión de esa instancia puede quedar suficientemente validada la citada función.

\section{El plano normativo de la función del bienestar colectivo}

El plano normativo de la función de bienestar colectivo, supone que la misma debe contribuir a la construcción de un marco de significados que favorezca el desarrollo de un lenguaje moral de cooperación. Es fácil percibir que este plano, aunque posee una estructura de justificación diferente de los anteriores, se encuentra inexorablemente ligado a los mismos, y contribuye junto con ellos a plantear una visión integrada del problema ético en la construcción de la función de bienestar colectivo.

Según se ha visto, a pesar de que el problema de distribución puede ser resuelto en el escenario de los juegos mediante procesos interactivos que logren la elaboración de un marco insesgado, esto no es garantía suficiente de que dicho marco sea el más valioso desde el punto de vista de la ética social. Para decidir acerca de este punto es necesario establecer qué debe entenderse como socialmente más valioso, y qué relación guarda ese algo con el contenido de la distribución que la función de bienestar realiza. 
Si admitimos que la condición básica para la existencia de la vida social reposa en la aceptación por parte de los individuos de restricciones racionales a sus intereses o preferencias, podemos asimismo admitir que la cooperación (entendida como autorrestricción racional) es un valor clave para la vida social, y merece ser estimulado y desarrollado. Sin embargo, se ha considerado siempre que la cooperación es un oficio difícil y riesgoso, razón por la cual la forma de las relaciones entre los miembros de una sociedad se ve muy bien reflejada en la estructura del dilema de los prisioneros. Este es un juego cuyo punto de equilibrio es no-cooperativo, y expresa claramente las dificultades que debe enfrentar un miembro de la sociedad cuando desea cooperar [Barragán/90].

Estas dificultades se derivan fundamentalmente del hecho de que la restricción racional de los intereses individuales puede tener como resultado indeseable la explotación de quien efectúa esa concesión. La desconfianza a ser víctima de tal explotación, como así también lo duro que resulta a veces vencer la tentación de aprovecharse indebidamente de las concesiones de los otros actores, son los principales obstáculos que encuentran la cooperación. Para derrotar tales obstáculos sin tener que apelar a soluciones autoritarias, es necesario desplazar el punto de equilibrio a la casilla cooperativa, y esto sólo se logra mediante la distribución apropiada de los beneficios sociales derivados de las restricciones a los beneficios individuales. Cuando lo que un individuo concede en términos de autolimitación al propio interés se ve compensado con lo que él recibe como participación en el beneficio colectivo, su deseo de conservar el juego social crece naturalmente.

Si consideramos estos puntos, cuando en una función de bienestar colectivo se articulan las funciones de bienestar individual, conforme a un marco de juego, no sólo se están dando las reglas para resolver un conflicto de intereses antagónicos mediante una cierta distribución, sino que también de acuerdo al modo en que esa distribución es realizada, se favorece o desfavorece el uso de la cooperación como lenguaje social. Hay en este aspecto normativo de la función de bienestar colectivo un carácter docente que contribuirá a fortalecer o debilitar (según el caso) la confianza de los individuos en las reglas que constituyen el marco del juego social.

El afianzamiento de esa confianza no es una cuestión estrictamente psicológica, sino que se proyecta en el plano de la construcción de la función individual de utilidad. En efecto, si la probabilidad subjetiva que cada individuo asigna al hecho de 
que las autorrestricciones que él impone a sus utilidades y preferencias le serán compensadas con una adecuada participación en las utilidades sociales es alta, podrá racionalmente incluir estas últimas en su función de utilidad, y el «costo» de cooperar le resultará bajo y con mínimo riesgo. En caso contrario, su deseo de cooperar no sólo se verá psicológicamente desalentado, sino que la cooperación constituiría un comportamiento irracional en términos de su función de utilidades. Desde este punto de vista, el carácter normativo de la función de bienestar colectivo se dirige a producir un refinamiento en la construcción de la función de bienestar individual, y una ampliación del marco de la racionalidad individual.

Como puede verse, el aspecto normativo de la función de bienestar colectivo supone la aceptación de una determinada meta como socialmente valiosa. En nuestro caso hemos utilizado la cooperación (como autorrestricción racional de las propias utilidades y preferencias) por considerarla básica para la convivencia social, y prácticamente implícita en la definición de la misma. Sin embargo, ese valor tendrá que ser sometido a discusión crítica y enfrentamiento con otros valores alternativos para el caso de cada sociedad en particular, pero, en definitiva, sea cual fuere el elemento normativo que la función de bienestar colectivo tienda a desarrollar, si el mismo no es suficientemente analizado y justificado, no podremos hablar en propiedad de tal función como decisión racional en el entorno social.

\section{Conclusión}

La construcción de la función de bienestar colectivo es una tarea compleja, que debe ser abordada en tres planos diferentes: el instrumental, el distributivo, y el normativo. El primero se relaciona con la determinación de una regla de decisión formal que sea coherente con los principios generales de la racionalidad social e individual. El segundo concierne a la elaboración de principios que permitan una solución insesgada de los conflictos que surgen en toda distribución de bienes escasos. Finalmente el tercero se vincula con el desarrollo de un marco normativo que estimule el uso de la cooperación como lenguaje moral.

En relación al plano instrumental, el primer problema que debe resolverse es el relativo a las comparaciones interpersonales de utilidades. A este respecto considero que el Teorema de Arrow, tan frecuentemente invocado para justificar la imposibilidad de tales comparaciones, es sólo válido en el universo definido 
por Arrow que es extremadamente pobre desde el punto de vista informacional, y que en consecuencia no parece plausible en el entorno de las sociedades contemporáneas. Entre quienes han logrado soluciones aceptables al problema de las comparaciones interpersonales de preferencias encontramos a Rawls, quien se inclina por asumir (como Arrow) el carácter ordinal de las mismas, y llega a la solución apelando a un orden lexicográfico y a la regla de decisión maximin. Entre quienes aceptan el principio de cardinalidad de las preferencias de von Neumann, la solución de Harsanyi, que considera que la utilidad social es una función lineal de todas las utilidades individuales si se les asigna a cada una de éstas el mismo peso relativo, es a la vez coherente y poderosa, aunque a la hora de cualificar las preferencias a ser tenidas en cuenta, debe apelar al metacriterio de la «hipótesis de las preferencias bien informadas». La superioridad de la fórmula instrumental de Harsanyi radica en que permite intercambios sutiles que favorecen el logro de soluciones.

Tanto el desarrollo de Rawls como el de Harsanyi ponen en evidencia que el solo plano instrumental no agota el problema de construcción de la función de bienestar colectivo, ya que tratándose de una función que resuelve un problema de asignación de recursos escasos o de cargas sociales, es siempre mediadora en un conflicto de intereses. Por esa razón es necesario considerar dicha función en su plano distributivo. Este plano, ligado inexorablemente al antagonismo, se vincula con dos instancias diferentes: una, en la que se construyen las reglas que regularán el juego, y la otra que es la del logro de la solución, instancia esta que se cumple dentro del escenario del juego mismo. En ambas instancias se pone en evidencia la superioridad del enfoque de von Neumann, ya que el mismo permite mover los intercambios entre preferencias y preferencias, y aun entre valores y valores con el fin de hacer posible los acuerdos que permitan en un caso el logro del marco de reglas para el juego, y en el otro el logro de la solución al juego mismo.

Sin embargo, lo máximo que las herramientas propias de los juegos pueden ofrecernos en el plano distributivo, son soluciones insesgadas, que no son capaces por sí mismas de garantizarnos resultados socialmente valiosos; y éste es un punto del que la construcción de la función de bienestar colectivo debe hacerse cargo. Por esa razón, cobra especial importancia en la misma el que hemos llamado plano normativo, que se alimenta con el contenido de la solución distributiva.

En el plano normativo debe fincarse la discusión acerca del valor social que la función de bienestar colectivo tenderá a estimular 
y desarrollar. En este caso me inclino por privilegiar el valor de la cooperación, definida como la autolimitación racional de las propias utilidades. Así considerada la cooperación se ubica en el centro mismo de la idea de cohesión social y de la definición de vida social. Son bien conocidas las dificultades que debe afrontar un individuo para ejercer la cooperación en el entorno social; el modelo del dilema de los prisioneros las ha expuesto con gran claridad, como también ha hecho evidente el resorte que puede transformar el original juego -suma -cero -no- cooperativo, en un juego suma -cero -cooperativo, disolviendo el dilema. La clave del mecanismo radica en cómo se efectúa la distribución en el marco de la función de bienestar colectivo. Si sólo contamos con reglas de juego insesgadas pero que pueden ser de alguna manera burladas, o dan lugar a formas sistemáticas de explotación, no habrá manera de estimular la cooperación, ya que si la probabilidad de obtener una participación en los beneficios sociales es muy baja, no sería coherente con la idea de racionalidad el cooperar. Tomando esto en consideración, cuando se realiza la distribución mediante la construcción de la función de bienestar colectivo, no sólo se resuelve un problema de asignación concreta de recursos, sino también se define el valor social que se pretende estimular. En este aspecto, la función de bienestar colectivo considerada como una decisión racional es una poderosa herramienta para el desarrollo del lenguaje moral de una sociedad determinada.

\section{BIBLIOGRAFÍA}

Arrow, K., Social Choice and Individual Values, J. Willey, Nueva York, 1951.

--, «A Difficulty in the Concept of Social Welfare», Journal of Political Economy, 58, 1950.

Axelrod, R., The Evolution of Cooperation, Basic Books, Nueva York, 1984.

Barragán, J., «Las Reglas de la Cooperación», Doxa, 6, 1989.

-, «Racionalidad Cooperativa», en Racionalidad Cientifica, Racionalidad Práctica y Racionalidad Teológica, E. Placenza (ed.), Caracas, 1989. 
-,«Bargaining and Uncertainty», en Proceedings de Logica-Informatica-Diritto, Firenze, 1989.

-, Hipótesis Metodológicas, Ed. Jurídica Venezolana, 1983.

Brandt, R., A Theory of the Good and the Right, Clarendon Press, Oxford, 1979.

Dworkin, R., «DeFunis v. Sweatt», en Equality and Preferential Treatment, T. Nagel (ed.), Princeton University, 1977.

Gauthier, D., Morals by Agreement, Oxford University Press, 1986.

Griffin, J., Well-beeing Its meaning, measurement and moral importance, Clarendon Press, Oxford, 1986.

Hare, R. M., Moral Thinking, Clarendon Press, Oxford, 1981.

Harsanyi, J., Decision and Game Theoretic Models in Utilitarian Ethics, University of California at Berkeley, 1988.

--, Equality, Responsability and Justice, University of California at Berkeley, 1990.

Kalal, E.; M. Sorodinski, «Other Solutions to Nash’s Bargaining Problern», Econométrica, vol. 43,3, 1975.

Kolnai, A., Ethics, Values and Realty, Hackett Pub. Co. Indianápolis, 1978.

Nash, J., «The Bargaining Problem», Econométrica, 18, 1950.

Rawls, J., A Theory of Justice, Oxford, University Press, 1972.

V. Neurnann, J.; O. Morgenstern, The Theory of Games and Economic Behavior, Willey, Nueva York, 1964 (c. 1944).

Walzer, M., Spheres of Justice, Basic Books, N. Y., 1983. 